\title{
Amorphophallus (Araceae) nouveaux d'Afrique centrale
}

par

\author{
F. Malaisse \& P. Bamps (*)
}

Abstract. - Description of three new species of Amorphophallus Blume ex Decne., two from Zaire and one from Burundi.

Amorphophallus lewallei Malaisse \& Bamps sp. nov.; affinis $A$. goetzei (Engl.) N.E. Br. sed spadicis sterili appendice perspicue breviore dimidioque superiore dense verrucosa (appendice omnino laevi in $A$. goetzei) atque ovario uniloculari (biloculari in A. goetzei) valde differt. - Fig. 2.

Plante vivace, totalement glabre, à tubercule souterrain, semi-globuleux, à sommet aplati et portant de nombreuses racines, de 7-9 $\mathrm{cm}$ de diam. et $3 \mathrm{~cm}$ de haut. Feville solitaire, connue seulement à l'état jeune (à l'époque de la floraison); pétiole de $16 \mathrm{~cm}$ de long, entouré d'un cataphylle de $16 \mathrm{~cm}$ de long et 1,4 $\mathrm{cm}$ de large, parsemé de petites incrustations blanchâtres; limbe palmatiséqué en 3 segments découpés en lanières d'abord étroites, puis devenant plus larges; nervures latérales obliques-ascendantes et parallèles; nervation tertiaire lâche et peu apparente. Inflorescence solitaire; pédoncule de 4-8 $\mathrm{cm}$ de long et 4-5 $\mathrm{mm}$ de diam., sous-tendu par 2 cataphylles de couleur paille, pouvant atteindre $15 \mathrm{~cm}$ de long et $3,5 \mathrm{~cm}$ de large, à face interne couverte de petites incrustations orangées, devenant plus grandes vers la base; spathe \pm urcéolée, atteignant $11 \mathrm{~cm}$ de longueur totale et $16 \mathrm{~cm}$ de large, fendue jusqu'à la base, à nombreuses nervures parallèles dans la moitié inférieure, plus étalées et plusieurs fois bifurquées

(*) F. Malaisse, Faculté des Sciences agronomiques, U.R. Ecologie, Passage des Déportés 2, B-5030 Gembloux (Belgique); P. Bamps, Jardin botanique national de Belgique, Domaine de Bouchout, B-1860 Meise (Belgique). - Manuscrit déposé le 7 octobre 1992. 
dans la partie supérieure; pseudo-tube subglobuleux, vert extérieurement, violet à mauve intérieurement, de $4-5 \mathrm{~cm}$ de hauteur et $5-6 \mathrm{~cm}$ de diam., lisse extérieurement, orné de nombreuses petites excroissances intérieurement; limbe évasé et tronqué obliquement, vert et lisse sur les 2 faces, à surface ondulée longitudinalement et pourvue de nombreuses petites incrustations blanches; spadice creux intérieurement, vert jaunâtre, de $11-15 \mathrm{~cm}$ de long, partie 9 basale, cylindrique, grise, de 7-20 $\mathrm{mm}$ de haut et 7-9 $\mathrm{mm}$ de diam. ; partie $\sigma^{7}$ non directement contiguë (séparée de la partie $q$ par un espace lisse de 1-2 mm), ovoïde, jaune, de $20-25 \mathrm{~mm}$ de haut et $15-16 \mathrm{~mm}$ de diam. ; appendice

cm stérile fusiforme, de 8-9 de long et $7 \mathrm{~mm}$ de diam. vers la base, lisse dans la moitié inférieure, couvert dans la moitié supérieure d'excroissances verruqueuses très denses et pouvant atteindre $2 \mathrm{~mm}$ de hauteur. Fleurs $O^{7}$ nombreuses et contiguës; étamines d'environ $1,5 \mathrm{~mm}$ de large, à 2 thèques à déhiscence apicale. Fleurs 9 non ou à peine contiguës; ovaire d'environ $2,5 \mathrm{~mm}$ de haut et $1,5 \mathrm{~mm}$ de diam., à 1 loge 1-ovulée; placentation basale; stigmate subsessile et discoïde. Fruits non connus.

Burundi : Prov. Buhanza, vallée de la Katurungu, Kihunga, alt. 900 m, savane sous Acacia, nov. 1969 (fl. \& fe. juvén.), Lewalle 4009 (BR holotype, K iso-); ibid., Kagongwe, alt. 900 m, savane, nov. 1972 (fl.), Reekmans 2133 (BR).

Amorphophallus mullendersii Malaisse \& Bamps sp. nov.; affinis $A$. teuszii (Engl.) N.E. Br. spatha produnde lobata sed spathae lobis 6 versus 3 stigmate convexo versus concavo spadicisque appendice quam spatha multo longiore versus leviter breviore valde differt. Fig. $1 \& 2$.

Plante vivace, totalement glabre, à tubercule souterrain, globuleuxaplati, de $6 \mathrm{~cm}$ de diam. et $2,5 \mathrm{~cm}$ de haut. Feuille solitaire, paraissant après la floraison, connue seulement par un dessin fragmentaire (voir fig. 1, d'après plante vivante observée en culture à Lubumbashi par F. Malaisse). Inflorescence solitaire; pédoncule de $8 \mathrm{~cm}$ de long et

Fig. 1. - Amorphophallus mullendersii Malaisse \& Bamps : A, plante entière $(\times 1 / 2)$; $B$, silhouette de la plante avec jeune inflorescence $(\times 1 / 4)$; $C$, fragment de feuille $(\times 1 / 2) ; D$, partie inférieure du spadice $(\times 11 / 2) ; \mathrm{E} \& \mathrm{~F}$, étamine vue de profil et d'au-dessus $(\times 8) ; \mathrm{G}, \mathrm{H}, \mathrm{I} \& \mathrm{~J}$, pistil vu latéralement, d'au-dessus, en coupe transversale et en coupe longitudinale (× 6). - D'après Malaisse 11911 (A, B, D-J) et plante vivante observée en culture (C). 


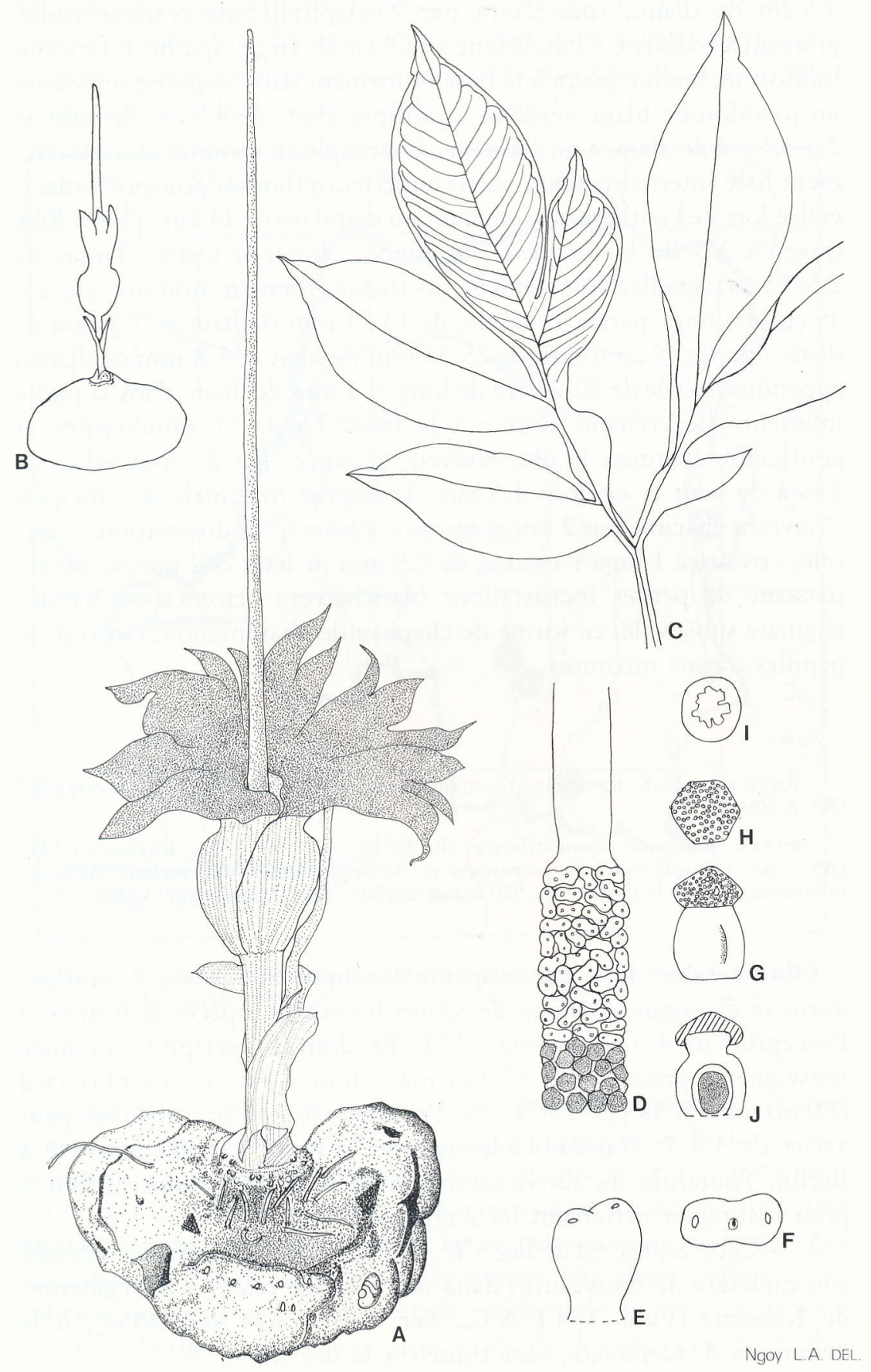


3-5 cm de diam., sous-tendu par 2 cataphylles de couleur paille, pouvant atteindre $6,5 \mathrm{~cm}$ de long et $0,7 \mathrm{~cm}$ de large ; spathe \pm infundibuliforme, fendue jusqu'à la base et formant dans sa partie inférieure un pseudotube blanc verdâtre à pourpre clair, de $4,5 \mathrm{~cm}$ de haut et 2,5-3,5 cm de diam. à mi-hauteur, \pm étranglé au sommet et complètement lisse intérieurement; partie supérieure (limbe) pourpre violacé, étalée lors de l'anthèse et atteignant un diamètre de $13 \mathrm{~cm}$, à bord lobé (jusqu'à 2/3 de la longueur du limbe); lobes 6 , ovales, larges de 2,5-3,5 M1 ; spadice longuement fusiforme, pourpre noirâtre, de 25$35 \mathrm{~cm}$ de long; partie $Q$ basale, de $13-21 \mathrm{~mm}$ de haut et $7-8 \mathrm{~mm}$ de diam.; partie $\sigma^{\text {T }}$ contiguë, de $25-35 \mathrm{~mm}$ de haut et 7-8 $\mathrm{mm}$ de diam.; appendice stérile de $20-29 \mathrm{~cm}$ de long et $5 \mathrm{~mm}$ de diam. dans la partie inférieure, légèrement rétréci à la base. Fleurs $\sigma^{7}$ nombreuses et contiguës; étamines le plus souvent groupées par 2, obovoïdes, de $2 \mathrm{~mm}$ de haut et environ $1,5 \mathrm{~mm}$ de largeur maximale, à 2 thèques s'ouvrant chacune par 2 pores apicaux. Fleurs $q$ en disposition \pm spiralée; ovaire à 1 loge 1 -ovulée, de $2,5 \mathrm{~mm}$ de haut et $2 \mathrm{~mm}$ de diam., parsemé de petites incrustations blanchâtres; placentation basale; stigmate subsessile, en forme de chapeau de champignon, couvert de papilles. Fruits inconnus.

\section{ZAÏRE :}

Kasai oriental : Kambaye, disséminé près de l'eau, sept. 1930 (fl.), Quarré 2040 (BR holotype).

ShabA: Kaniama, savane arborée, alt. 900 m, sept. 1947 (fl.), Mullenders 1048 (BR); riv. Lufupa, entre Kyamasumba et Busanga, savane sur rochers en pente moyenne, près de la rivière, alt. $920 \mathrm{~m}$, sept. 1981 (fl.), Malaisse 11911 (BR).

Observations: 1. - Par sa spathe découpée en 6 lobes, A. mullendersii se distingue aisément de toutes les autres espèces africaines, à l'exception de A. teuszii (Engl.) N.E. Br. dont la description originale renseigne la présence de 3 lobes mais dont il est signalé plus tard (Pflanzenreich 48 [IV-23C]: 73. 1911) que le nombre de lobes peut varier de 3 à 7 , vraisemblablement sur base du spécimen cultivé à Berlin. Toutefois, les autres caractères de la diagnose sont suffisants pour distinguer nettement les 2 espèces.

2. - Cette espèce est dédiée à $W$. Mullenders qui le premier a décelé son caractère de nouveauté; dans son ouvrage relatif à la végétation de Kaniama (Publ. I.N.E.A.C., Sér. Scient. 62: 463. 1954), il la dénomma $A$. kaniamae, sans toutefois la décrire. 
Amorphophallus petitianus Malaisse \& Bamps sp. nov.; affinis $A$. goetzi (Engl.) N.E. Br. sed spatha parviore haud constricta, spadicis foeminea masculeaque in diametro subaequalibus et stigmate discoideo nec globoso valde differt. - Fig. 2.

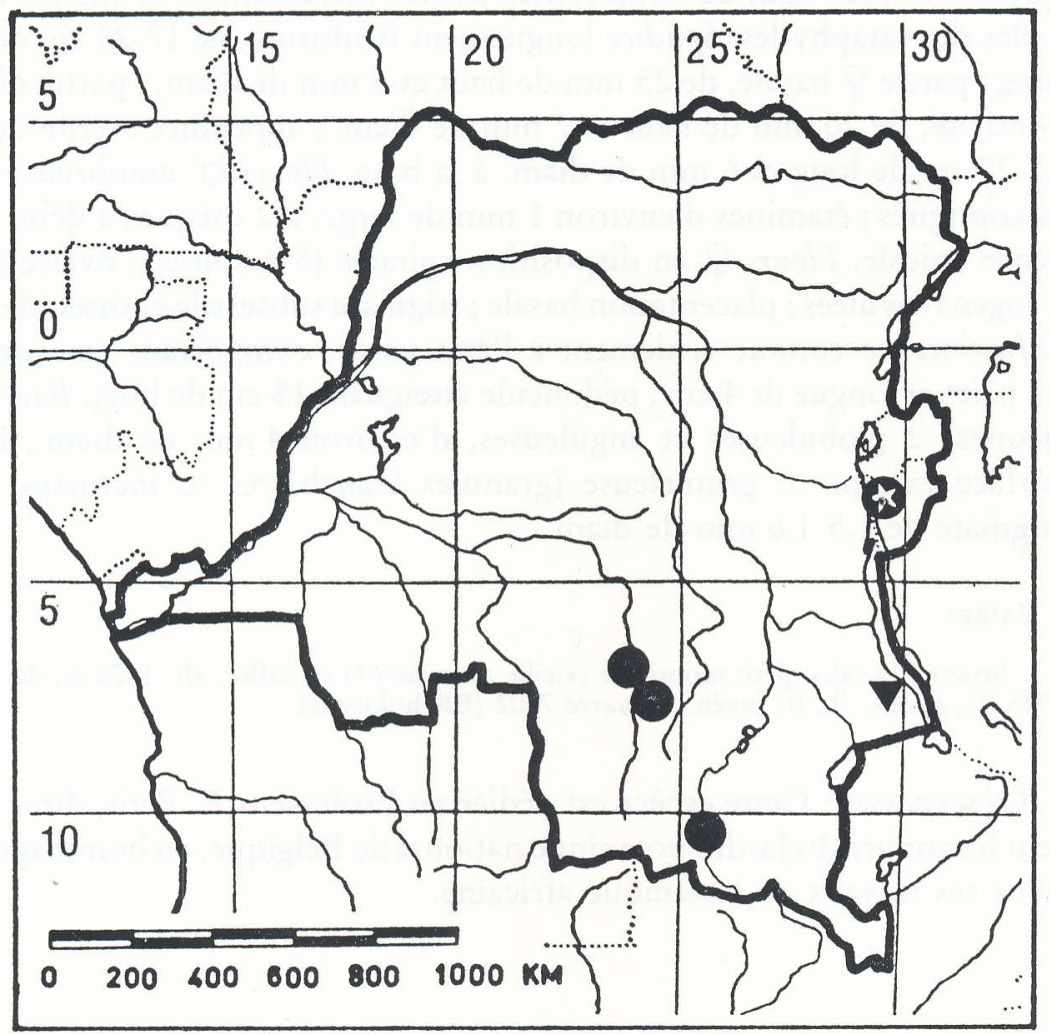

Fig. 2. - Distribution d'Amorphophallus lewallei (étoile blanche dans rond noir), A. mullendersii (ronds noirs) et $A$. petitianus (triangle noir renversé).

Plante vivace, totalement glabre, à tubercule souterrain. Fenille solitaire, connue seulement à l'état jeune (à l'époque de la floraison); pétiole de $12 \mathrm{~cm}$ de long, entouré d'un cataphylle de $10 \mathrm{~cm}$ de long et environ $1 \mathrm{~cm}$ de large, pourvu de nombreuses petites incrustations surtout visibles sur la face interne; limbe palmatiséqué en 3 segments découpés en lanières étroites et finement réticulées. Inflorescence solitaire, apparaissant avant la feuille; pédoncule de $8-12 \mathrm{~cm}$ de long et $3 \mathrm{~mm}$ de diam., sous-tendu par 2 cataphylles plus courts et plus 
larges que celui de la feuille; spathe \pm obconique, de $10-11 \mathrm{~cm}$ de long et $3,5 \mathrm{~cm}$ de diam., fendue jusqu'à la base mais formant un pseudotube d'environ $5 \mathrm{~cm}$ de haut, à bord entier à ondulé, pourvue dans le $1 / 4$ inférieur de la face interne de nombreuses petites excroissances verruqueuses et plus haut de nombreuses petites incrustations similaires à celles des cataphylles. Spadice longuement fusiforme, de $17-25 \mathrm{~cm}$ de long; partie $Q$ basale, de $25 \mathrm{~mm}$ de haut et $8 \mathrm{~mm}$ de diam.; partie $O^{7}$ contiguë, de $20 \mathrm{~mm}$ de haut et $7 \mathrm{~mm}$ de diam.; appendice stérile de $13-20 \mathrm{~cm}$ de long et $6 \mathrm{~mm}$ de diam. à la base. Fleurs $\sigma^{7}$ nombreuses et contiguës; étamines d'environ $1 \mathrm{~mm}$ de large, à 2 thèques à déhiscence apicale. Fleurs $q$ en disposition spiralée (5-6 spires); ovaire à 2 loges 1-ovulées; placentation basale; stigmate subsessile et discoïde. Infrutescence connue seulement à l'état jeune, comportant environ 52 baies et longue de $4 \mathrm{~cm}$; pédoncule atteignant $15 \mathrm{~cm}$ de long. Baies (jeunes) \pm globuleuses et anguleuses, d'environ $4 \mathrm{~mm}$ de diam., à surface externe \pm grumeleuse (granules blanchâtres \pm incrustés); stigmate de 1,5-1,6 $\mathrm{mm}$ de diam.

\section{ZAÏRE :}

SHABA : Lusaka, petit monticule (vieille termitière ?) en vallée, alt. $1200 \mathrm{~m}$, déc. 1945 (fe. juvén., fl., fr. juvén.), Quarré 7502 (BR holotype).

Observation: Cette espèce est dédiée au Professeur E. Petit, directeur honoraire du Jardin botanique national de Belgique, en hommage pour ses travaux de botanique africaine. 